\title{
Breast cancer mortality trends in two areas of the province of Florence, Italy, where screening programmes started in the 1970s and 1990s
}

\author{
G Gorini*,', M Zappa ${ }^{2}$, G Miccinesi ${ }^{2}$, E Paci ${ }^{2}$ and A Seniori Costantini' \\ 'Unit of Occupational and Environmental Epidemiology, Centre for Study and Prevention of Cancer (CSPO), Via di S. Salvi, Florence I 2-50 I 35, Italy; ${ }^{2}$ Unit \\ of Clinical and Descriptive Epidemiology, Centre for Study and Prevention of Cancer (CSPO), Via di S. Salvi, Florence 12-50 I 35, Italy
}

We compared breast cancer mortality rates in the period 1985-2000 in two areas of the province of Florence, Italy, where breast cancer screening programmes started in the 1970s (early screening (ES) area) and in 1990s (late screening (LS) area). The overall age-standardised mortality decreased in the whole period by $40.9 \%$ in the ES area $(P<0.001)$, and by $11.3 \%$ in the LS area $(P=0.030)$. Significant decreases in the ES area were detected in groups aged $45-54$ years $(61.1 \% ; P=0.018)$ and $65-74$ years (44.7\%; $P=0.049)$, whereas in the LS area no significant decrease was detected in any age group. The relatively low compliance in the first years of the programme in both areas, and the long enrolment period in the LS area could have reduced the effect on mortality. Our findings suggest that the drop in mortality in the ES area (4I\%) could be explained by both service screening and better care. The slight decrease in mortality in the LS area (I I\%) could be mainly due to better care. A reduction of about $30 \%$ is attributable to screening in the ES area over the period 1985-2000.

British Journal of Cancer (2004) 90, I780- 1783. doi:I0.1038/sj.bjc.6601744 www.bjcancer.com

Published online 23 March 2004

(C) 2004 Cancer Research UK

Keywords: screening; breast; early diagnosis; mortality

Mammographic screening has been shown to reduce breast cancer mortality rates (Vainio and Bianchini, 2002). Several studies in northern European countries (Hermon and Beral, 1996; Hakama et al, 1997; Van den Akker-van Marle et al, 1999; Blanks et al, 2000; Jonsson et al, 2001; Botha et al, 2003; Otto et al, 2003; Tabar et al, 2003) have reported a decrease in breast cancer mortality where nationwide screening programmes have been implemented. There has, however, been considerable debate concerning the relative contributions of screening and of improved therapy to the observed trends (Carnon et al, 1996; Blanks et al, 2000; Peto et al, 2000).

At the beginning of the 1970s in 23 rural municipalities of the province of Florence, Italy (early screening area (ES area) among about 70000 resident women aged 25 or over, $18 \%$ of women of the province, based on 1991 Census), a mammographic screening programme was started; until 1989, women in the $40-69$ year age group were invited, whereas from that year onwards the target population was restricted to age group 50-69 years. The number of mammograms performed in the ES area was 8000-9000 per year. The efficacy of the programme on breast cancer mortality in the ES area has been evaluated for the period 1977-1984 by means of case-control studies (Palli et al, 1986, 1989), and by using early indicators of efficacy (Paci et al, 1990). After 1990, when the screening for women aged 50-69 years started in the rest of the province, including the city of Florence (late screening area: LS

*Correspondence: Dr G Gorini; E-mail: g.gorini@cspo.it

Received 26 November 2003; revised 2 February 2004; accepted 2 February 2004; published online 23 March 2004 area), the number of mammograms in the whole province increased to 28000-29000 per year (Barchielli et al, 1999).

In this study, we have compared breast cancer mortality rates in the period 1985-2000 in the ES and LS areas.

\section{MATERIALS AND METHODS}

\section{Sources of data}

Data on breast cancer mortality for the period 1985-2000 were obtained from the Tuscan Regional Mortality Register (Chellini et al, 2002), which collects death certificates of the residents of Tuscany. Incident breast cancer cases for the period 1985-1999 were obtained from the Tuscan Cancer Register, a member of the International Association of Cancer Registries (Parkin et al, 2002).

\section{Statistical analysis}

Adjusted mortality and incidence rates using direct standardisation to the European Standard Population, and age-specific (35$44,45-54,55-64,65-74,75-84$ and $\geqslant 85$ years) incidence and mortality rates with $95 \%$ confidence intervals (95\% CI) were calculated.

In order to calculate the estimated percent change (EPC) in rates for the whole period of observation, trends in mortality and incidence based on annual data were examined using a log-linear regression model, and the year of death as a continuous variable. 


\section{RESULTS}

The age-adjusted mortality rate in the ES area (Figure 1) was 22.4 per 100000 women in 1985 (95\% CI: $13.6-31.2 ; 27$ deaths), 31.8 in 1991 (95\% CI: $21.0-42.6$; 38 deaths) and then decreased to 10.9 in 2000 (95\% CI: 5.4-16.5; 19 deaths). In the LS area, the corresponding age-standardised mortality rates were more stable with only a slight decrease over time: in 198530.1 per 100000 women (95\% CI: $25.5-34.7 ; 186$ deaths); 27.7 in 2000 (95\% CI: $23.3-32.1 ; 190$ deaths). In the ES area, age-specific mortality rates decreased steadily from 1985 to 1986 until 1999-2000 in all age groups, except for $85+$ age group (Figure 2). The age-specific rates in the LS area were more stable (Figure 3).

In the ES area, incidence was 76.9 per 100000 women in 1985 (95\% CI: 59.6-94.1), 96.7 in 1989 (95\% CI: 77.6-115.8), 62.5 in 1993 (95\% CI: $47.9-77.1)$ and 110.6 in 1999 (95\% CI: 90.8-130.4). In the LS area, incidence rates were higher: 91.1 per 100000 women in 1985 (95\% CI: 82.6-99.6), 112.1 in 1993 (95\% CI: $102.9-121.4$ ) and 113.9 in 1999 (95\% CI: $104.5-123.4)$.

In addition to visual inspection, we measured EPCs in rates over the whole period (Table 1). In the period 1985-2000, the overall age-standardised mortality decreased by $40.9 \%$ in the ES area $(P<0.001)$, and by $11.3 \%$ in the LS area $(P=0.030)$. Significant decreases in the ES area were detected in age groups $45-54$ years $(61.1 \% ; P=0.018)$ and $65-74$ years $(44.7 \% ; P=0.049)$. In the $75-$ 84 age group, the decrement was $45.2 \%(P=0.053)$. In the LS area, no significant decreases were detected in any age group.

In the period 1985-1999, the overall age-standardised incidence increased by $12.9 \%$ in the ES area $(P=0.190)$, and by $14.2 \%$ in the LS area $(P<0.001)$ (Table 1$)$. In the ES area, no significant increases or decreases were detected in any age groups; in the LS area, where the prevalence screening was carried out in the period 1990 - 1994, significant increases were detected in groups aged 5564 years $(21.0 \% ; P=0.009)$ and 85 years and over $(45.1 \%$; $P=0.012)$.

\section{DISCUSSION}

Decreases in breast cancer mortality have been variously reported following the introduction of mammographic screening on a regional level (Tornberg et al, 1994; Quinn and Allen, 1995; Garne et al, 1997; Barchielli and Paci, 2001; Broeders et al, 2001; Tabar et al, 2001; Duffy et al, 2002). Randomised prospective trials indicate that benefits in terms of cumulative breast cancer mortality start to emerge 4-10 years after randomisation (Nyström et al, 2002). We observed a similar trend in the mortality rates in the two areas up to 1993 , although the mortality

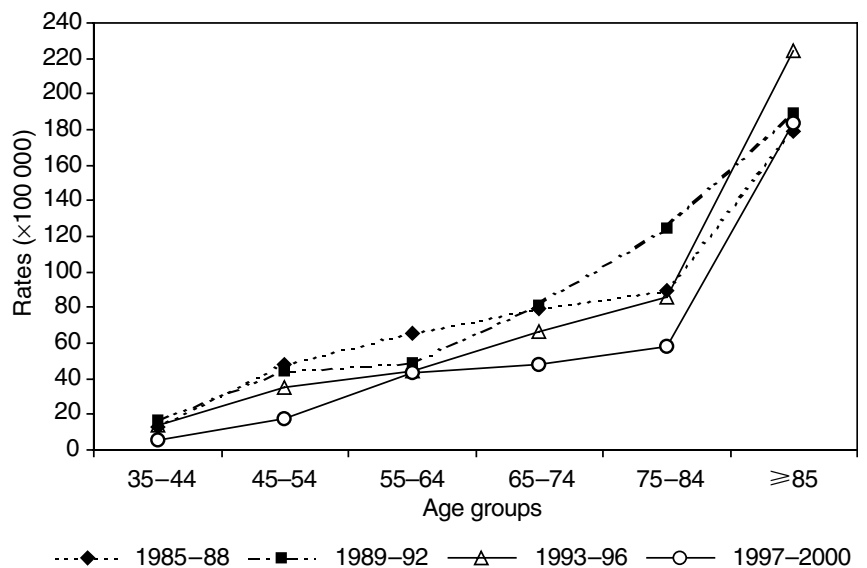

Figure 2 Age-specific mortality rates in the period 1985-2000 in the ES area of the province of Florence, Italy.

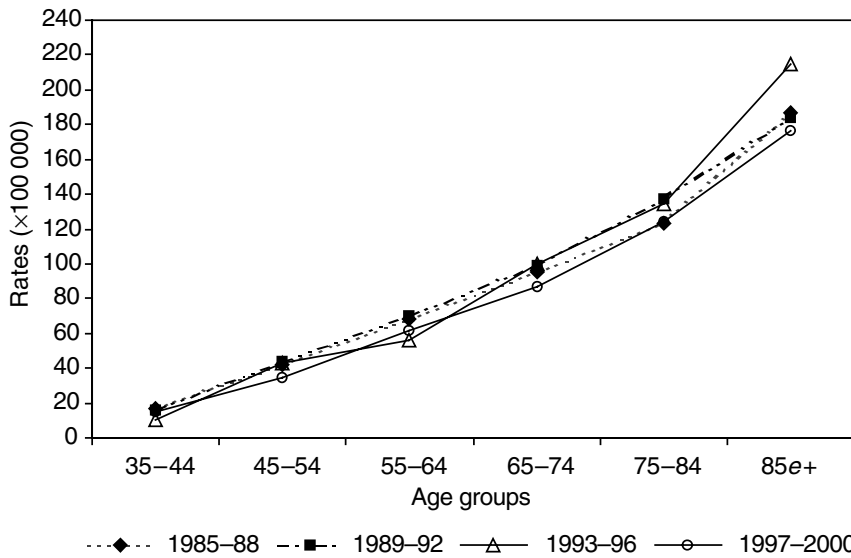

Figure 3 Age-specific mortality rates in the period 1985-2000 in the LS area of the province of Florence, Italy.

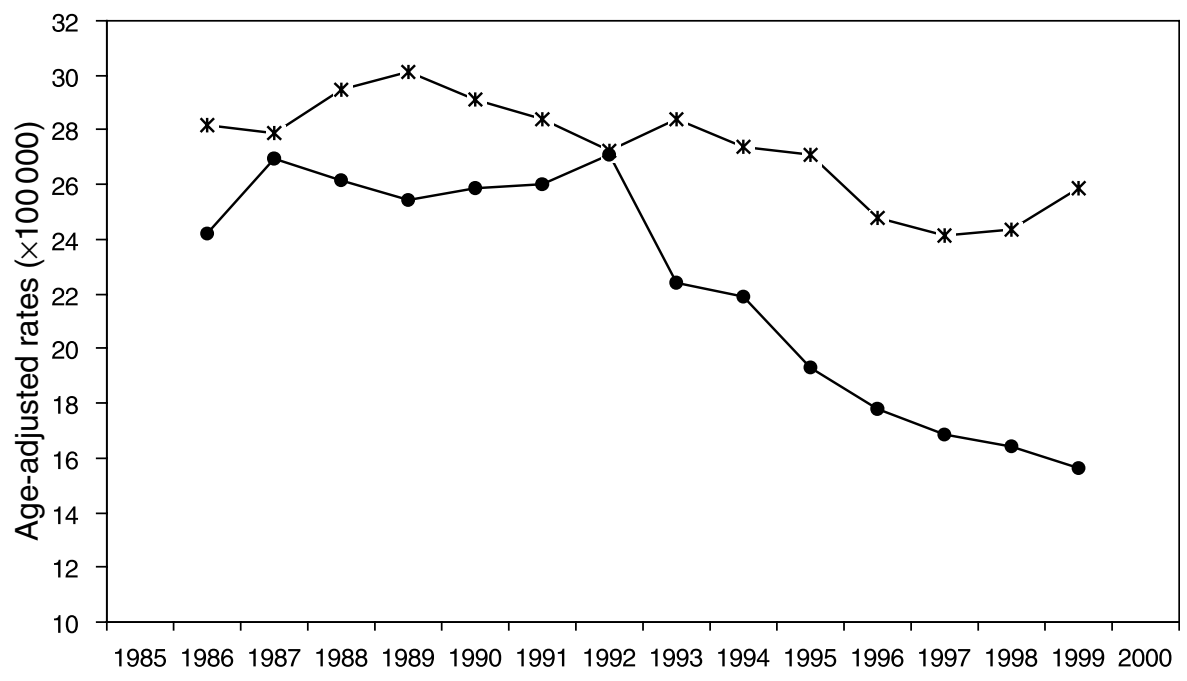

$-*$ - Late screening area $\rightarrow$ Early screening area

Figure I Age-adjusted (European population) breast cancer mortality rates in the ES area and in the LS area. Period 1985-2000, 3-year moving averages. 
Table I Estimated percentage change (EPC) and 95\% confidence intervals (95\% Cl) of mortality rates (1985-2000) and incidence rates (I985- I999) in Early Screening area and in Late Screening area

\begin{tabular}{|c|c|c|c|c|c|c|c|c|}
\hline \multirow[b]{3}{*}{ Age (years) } & \multicolumn{4}{|c|}{ Mortality (1985-2000) } & \multicolumn{4}{|c|}{ Incidence (1985- 1999) } \\
\hline & \multicolumn{2}{|c|}{ ES area } & \multicolumn{2}{|c|}{ LS area } & \multicolumn{2}{|c|}{ ES area } & \multicolumn{2}{|c|}{ LS area } \\
\hline & EPC (\%) & $95 \% \mathrm{Cl}$ & EPC (\%) & $95 \% \mathrm{Cl}$ & EPC (\%) & $95 \% \mathrm{Cl}$ & PC (\%) & $95 \% \mathrm{Cl}$ \\
\hline $35-44$ & -53.4 & $-87.4 ;+72.9$ & -21.7 & $-55.2 ;+36.9$ & +12.6 & $-33.8 ;+91.6$ & +17.0 & $-6.1 ;+45.8$ \\
\hline $45-54$ & -61.1 & $-82.2 ;-14.9$ & -18.4 & $-41.3 ;+13.5$ & +31.2 & $-8.3 ;+87.9$ & +7.1 & $-5.6 ;+21.5$ \\
\hline $55-64$ & -41.6 & $-69.6 ;+12.5$ & -15.7 & $-35.3 ;+9.9$ & +5.5 & $-24.8 ;+48.2$ & +21.0 & $+4.8 ;+39.8$ \\
\hline $65-74$ & -44.7 & $-69.8 ;-0.3$ & -10.8 & $-29.7 ;+13.3$ & -2.9 & $-31.5 ;+37.8$ & +8.4 & $-6.0 ;+24.9$ \\
\hline $75-84$ & -45.2 & $-70.9 ;+3.5$ & -5.1 & $-25.5 ;+20.9$ & +28.2 & $-14.6 ;+92.4$ & +15.9 & $-2.1 ;+37.3$ \\
\hline$\geqslant 85$ & +12.0 & $-46.2 ;+133.2$ & -5.7 & $-31.7 ;+30.0$ & +6.1 & $-47.5 ;+114.6$ & +45.1 & $+8.5 ;+94.1$ \\
\hline $35-85+$ & -36.0 & $-52.1 ;-14.4$ & -3.0 & $-13.8 ;+9.2$ & +13.3 & $-4.0 ;+33.8$ & +19.0 & $+11.4 ;+27.1$ \\
\hline Age adjusted & -40.9 & $-55.9 ;-20.9$ & -11.3 & $-21.3 ;-0.2$ & +12.9 & $-4.4 ;+33.3$ & +14.2 & $+6.9 ;+22.1$ \\
\hline
\end{tabular}

$E P C=$ estimated percentage change; $95 \% \mathrm{Cl}=95 \%$ confidence intervals; $E S$ area = early screening area; $\mathrm{LS}$ area $=$ late screening area

rates in the ES area were lower than those in the LS area. Afterwards a marked fall occurred in ES area, whereas only a slight decrease was observed in the LS area. The relatively low compliance in the first years of the programme in both areas (about 55\%) and the long enrolment period (on average 2.5 years) in the LS area (Paci et al, 2002a,b) could have diluted the effect so that a decrease in mortality rates was observed only after a longer interval.

Our findings suggest that the drop in mortality in the ES area (41\%) could be explained by both service screening and better care. The slight decrease in mortality in the LS area $(11 \%)$ could be explained by better care, while service screening of the period $1990-2000$ in the LS area could have been responsible for only a small number of deaths occurring in the target population. In fact, it has been estimated that in the city of Florence, the breast cancer mortality reduction at ages 50-74 years attributable to screening was $3.2 \%$ over the period 1990-1999 (Paci et al, 2002a). Thus, allowing a $11 \%$ reduction due to better care, a reduction of about $30 \%(41-11 \%)$ is attributable to screening in the ES area over the period 1985-2000. These findings are consistent with the mortality reduction of about $25 \%$ in screened populations shown by randomised trials (Vainio and Bianchini, 2002).

The decrease in mortality at ages $45-54$ years in the ES area could be related to the involvement in mammographic screening of women aged 40-49 years in the ES area until 1993. In 1985-1993, $33 \%$ of mammograms in the ES area (about 2700 per year) were performed on women aged 40-49 years. No mortality reduction would be expected from screening in the younger age group (3544 years) or in the 85 years and over age group.

In evaluating these results, it must be borne in mind that the ES area, where the incidence rates were lower, was a less urbanised area during the period of observation. The significant increase in incidence recorded only in the LS area (of 14.2\%), particularly in women aged 55-64 years (21.0\%), could be explained by earlier detection. Increases in incidence have been recorded in other Western countries in relation to screening (Quinn and Allen, 1995; Garne et al, 1997; Persson et al, 1998; Chu et al, 1996; Rostgaard et al, 2001). In the ES area, no significant increase in incidence was detected. It is likely that an increase in incidence in the ES area should have occurred in the 1970s, as a result of screening.

Incidence trends in the ES and LS areas do not seem to explain the observed differences in mortality rates during the study period. The present results seem to confirm that service screening can reduce breast cancer mortality rates.

\section{ACKNOWLEDGEMENTS}

We thank Andrea Martini and Paola Piccini for statistical assistance, and Vanessa Bolejack for proofreading.

\section{REFERENCES}

Barchielli A, Paci E, Giorgi D (1999) Recent trends of in situ carcinoma of the breast and mammographic screening. Cancer Causes Control 10: $313-317$

Barchielli A, Paci E (2001) Trends in breast cancer mortality, incidence, and survival and mammographic screening in Tuscany, Italy. Cancer Causes Control 12: 249-255

Blanks RG, Moss SM, McGahan CE, Quinn MJ, Babb PJ (2000) Effect of NHS breast screening programme on mortality from breast cancer in England and Wales, 1990-8: comparison of observed with predicted mortality. BMJ 321: $665-669$

Botha JL, Bray F, Sankila R, Parkin DM (2003) Breast cancer incidence and mortality trends in 16 European countries. Eur J Cancer 39: $1718-1729$

Broeders MJ, Peer PG, Straatman H, Beex LV, Hendriks JH, Holland R, Verbeek AL (2001) Diverging breast cancer mortality rates in relation to screening? A comparison of Nijmegen to Arnhem and the Netherlands, 1969-97. Int J Cancer 92: $303-308$
Carnon A, Hole D, Gillis C, Brewster D (1996) Incidence of and mortality from breast cancer since introduction of screening. Several factors must have a role in improved figures. BMJ 312: 640

Chellini E, Giovannetti L, Gorini G, Sorso B, Fornai MG, Martini A, Lisi C, Querci A (2002) Morti per causa anno 2000. Registro Mortalità Regionale. Firenze: Regione Toscana

Chu KC, Tarone RE, Kessler LG, Ries LA, Hankey BF, Miller BA, Edwards BK (1996) Recent trends in U.S. breast cancer incidence, survival and mortality rates. J Natl Cancer Inst 88: 1571-1579

Duffy SW, Tabar L, Chen HH, Holmqvist M, Yen MF, Abdsalah S, Epstein B, Frodis E, Ljungberg E, Hedborg-Melander C, Sundbom A, Tholin M, Wiege M, Akerlund A, Wu HM, Tung TS, Chiu YH, Chiu CP, Huang CC, Smith RA, Rosen M, Stenbeck M, Holmberg L (2002) The impact of organized mammography service screening on breast carcinoma mortality in seven Swedish counties. Cancer 95: 458-469

Garne J, Aspergren K, Balldin G, Ranstam J (1997) Increasing incidence of and declining mortality from breast carcinoma. Trends in Malmo, Sweden. Cancer 79: 69-74 
Hakama M, Pukkala E, Heikkilä M, Kallio M (1997) Effectiveness of the public health policy for breast cancer screening in Finland: population based cohort study. BMJ 314: $864-867$

Hermon G, Beral V (1996) Breast cancer mortality rates are levelling off or beginning to decline in many western citites: analysis of time trends, age-cohort and age-period models of breast cancer mortality in 20 countries. Br J Cancer 73: $955-960$

Jonsson H, Nystrom L, Tornberg S, Lenner P (2001) Service screening with mammography of women aged 50-69 years in Sweden: effects on mortality from breast cancer. J Med Screen 8: $152-160$

Nyström L, Andersson I, Bjurstam N, Frisell J, Nordenskjöld B, Rutqvist LE (2002) Long-term effects of mammography screening: updated overview of the Swedish randomised trials. Lancet 359: 909-919

Otto SJ, Fracheboud J, Looman CWN, Broeders MJM, Boer R, Hendriks JHCL, Verbeek ALM, de Konig HJ, and the National Team for Breast Cancer Screening (2003) Initiation of populationbased mammography screening in Dutch municipalities and effect on breast-cancer mortality: a systematic review. Lancet 361: $1411-1417$

Paci E, Ciatto S, Buiatti E, Cecchini S, Palli D, Rosselli del Turco M (1990) Early indicators of efficacy of breast cancer screening programmes. Results of the Florence District Programme. Int J Cancer 46: $198-202$

Paci E, Giorgi S, Bianchi S, Vezzosi V, Zappa M, Crocetti E, Rosselli del Turco M (2002a) Assessment of the early impact of the population-based breast cancer screening in Florence (Italy) using mortality and surrogate measures. Eur J Cancer 38: $568-573$

Paci E, Duffy SW, Giorgi D, Zappa M, Crocetti E, Vezzosi V, Bianchi S, Rosselli E, Rosselli del Turco M (2002b) Quantification of the effect of mammographic screening on fatal breast cancers: the Florence Programme 1990-96. Br J Cancer 87: 65-69

Palli D, Del Turco MR, Buiatti E, Carli S, Ciatto S, Toscani L, Maltoni G (1986) A case-control study of the efficacy of a nonrandomized breast cancer screening program in Florence (Italy). Int J Cancer 38: $501-504$
Palli D, Rosselli del Turco M, Buiatti E, Ciatto S, Crocetti E, Paci E (1989) Time interval since last test in a breast cancer screening programme: a case-control study in Italy. J Epidemiol Commun Health 43: 241 - 248

Parkin D, Whelan SL, Ferlay J, Teppo L, Thomas DB (2002) Cancer Incidence in Five Continents, Vol. VIII, IARC Scientific Publication No. 155. Lyon, France: International Agency for Research on Cancer, World Heath Organization

Persson J, Bergstrom R, Barlow L, Adami HO (1998) Recent trends in breast cancer incidence in Sweden. Br J Cancer 77: 167-169

Peto R, Boreham J, Clarke M, Davies C, Beral V (2000) UK and USA breast cancer deaths down $25 \%$ in year 2000 at ages 20-69 years. Lancet 355: 1822

Quinn M, Allen E (1995) Changes in incidence and mortality from breast cancer in England and Wales since introduction of screening. BMJ 311: $1391-1395$

Rostgaard K, Vaeth M, Holst H, Madsen M, Lhynge E (2001) Age-periodcohort modeling for breast cancer incidence in the Nordic countries. Stat Med 20: $47-61$

Tabar L, Vitak B, Chen HH, Yen MF, Duffy SW, Smith RA (2001) Beyond randomized clinical trials: organized mammographic screening substantially reduces breast cancer mortality. Cancer 91: 1724-1731

Tabar L, Yen MF, Vitak B, Chen HHT, Smith RA, Duffy SW (2003) Mammography service screening and mortality in breast cancer patients: 20-year follow-up before and after the introduction of screening. Lancet 361: $1405-1410$

Tornberg S, Carstersen J, Hakyulinen T, Lenner P, Hatschek T, Lundgren B (1994) Evaluation of the effect on breast cancer mortality of populationbased mammography screening programmes. J Med Screen 1: $184-187$

Vainio H, Bianchini F (2002) Breast Cancer Screening, IARC Handbooks of Cancer Prevention, Vol. 7 Lyon, France: International Agency for Research on Cancer, World Heath Organization

Van den Akker-van Marle E, de Koning H, Boer R, van der Maas P (1999) Reduction in breast cancer mortality due to the introduction of mass screening in The Netherlands: comparison with the United Kingdom. J Med Screen 6: $30-34$ 